\title{
Disuse-Induced Enhancement of la Synaptic Transmission in Spinal Motoneurons of the Rat
}

\author{
T. Manabe, S. Kaneko, and M. Kuno \\ Department of Physiology, Kyoto University Faculty of Medicine, Kyoto 606, Japan
}

\begin{abstract}
Monosynaptic excitatory postsynaptic potentials (EPSPs) evoked in rat spinal motoneurons by stimulation of a muscle nerve are enhanced during the first few days after section of the muscle nerve before subsiding to subnormal levels. We have examined whether this biphasic alteration results from changes of different factors involved in the regulation of central synaptic function. We chronically blocked the conduction of the intact peripheral nerve with tetrodotoxin (TTX). Under this disuse condition, enhancement of monosynaptic EPSPs was fully expressed within $2 \mathrm{~d}$, and synaptic function remained elevated for at least $3 \mathrm{~d}$, even after resumption of the impulse activity following discontinuance of the TTX. The magnitude of synaptic enhancement and the minimal period of nerve block required for synaptic enhancement were both comparable to those observed following nerve section. Thus, the early synaptic enhancement produced by section of the peripheral nerve could be accounted for entirely by the deprivation of sensory impulse actlvity. However, a prolonged nerve block up to $6 \mathrm{~d}$ still maintained synaptic enhancement. This was in contrast with synaptic depression observed after the first 3-4 $d$ following section of the peripheral nerve. The late synaptic depression can thus be attributed to changes in some factors other than sensory impulse activity. Therefore, sensory impulse activity and the peripheral axonal continuity of sensory fibers appear to be 2 distinct factors for the regulation of central synaptic function.
\end{abstract}

Monosynaptic excitatory postsynaptic potentials (EPSPs) evoked in spinal motoneurons by stimulation of group Ia sensory fibers from a muscle are depressed following section of the muscle nerve in the cat (Eccles et al., 1959; Gallego et al., 1979; Goldring et al., 1980). This synaptic depression has been reported to begin 10-14 d after nerve section (Eccles et al., 1959; Gallego et al., 1979) and proceed slowly until the synaptic responses virtually disappear 6-8 months later (Goldring et al., 1980). The mechanism underlying this phenomenon was initially postulated to be due to prolonged disuse of the central synapses induced by the deprivation of impulse activity in the cut sensory input (Eccles et al., 1959). However, daily stimulation of the central stump of the cut sensory fibers failed to prevent the central synaptic depression (Gallego et al., 1979). Also, when the conduction of an intact muscle nerve was locally blocked with tetrodotoxin (TTX) for 2 weeks, monosynaptic EPSPs elicited in

\footnotetext{
Reccived Aug. 10, 1988; revised Nov. 25, 1988; accepted Nov. 30, 1988.

This work was supported by research grants from the Ministry of Education, Science and Culture of Japan.

Correspondence should be addressed to Motoy Kuno at the above address.

Copyright (C) 1989 Society for Neuroscience $0270-6474 / 89 / 072455-07 \$ 02.00 / 0$
}

motoneurons by stimulation of the muscle nerve were significantly enhanced (Gallego et al., 1979). Thus, the effects of severance of a peripheral nerve on central synaptic transmission are different from those observed after elimination of the sensory impulse activity. Plastic changes at Ia synapses on motoneurons induced by prolonged disuse are apparently characterized by enhancement, rather than by depression. However, it is not known how soon central synaptic enhancement may develop after elimination of sensory impulse activity with TTX. Recently, Gallego and Geijo (1987) have shown that a $2 \mathrm{~d}$ conduction block of preganglionic fibers with TTX is sufficient to increase the amplitude of EPSPs recorded from sympathetic ganglionic cells in the guinea pig. Furthermore, Miyata and Yasuda (1988) have reported that monosynaptic EPSPs evoked in motoneurons by muscle afferent volleys in the rat are transiently potentiated for the first few days after section of the muscle nerve before the synaptic responses eventually diminish. It is then possible that plastic enhancement at Ia synapses induced by the deprivation of sensory impulse activity may occur more quickly than has previously been suggested, i.e., 2 weeks (Gallego et al., 1979), and that the early potentiation of monosynaptic EPSPs observed after nerve section (Miyata and Yasuda, 1988) may reflect the effect of elimination of sensory impulses. The present study was devoted to testing these possibilities.

\section{Materials and Methods}

Preparation. Wistar rats, $28-38 \mathrm{~d}$ old, were anesthetized with pentobarbital sodium $(50 \mathrm{mg} / \mathrm{kg}$, i.p.). Chronic conduction block of the left sciatic nerve was achieved by subepineural insertion of a glass capillary filled with a TTX solution at the midthigh level in the manner described by Mills and Bray (1979) and Tsujimoto and Kuno (1988). Briefly, one end of a glass capillary $(0.75 \mathrm{~mm}$ o.d., $0.28 \mathrm{~mm}$ i.d.) was tapered and fire-polished to restrict its internal diameter to $15 \mu \mathrm{m}$ (total capillary length, $6-8 \mathrm{~mm}$ ). After filling the tube with a TTX solution, $80 \mathrm{~mm}$ (Sankyo Co.), the unrestricted end of the capillary was sealed with hematocrit tube sealant to allow leakage of the TTX solution only from the restricted end. Paralysis of the left hind leg occurred within $1 \mathrm{hr}$ after the capillary implantation, as evidenced by the disappearance of the toe-spreading reflex. The absence of this reflex was confirmed twice daily, and the conduction block was maintained for $1-6 \mathrm{~d}$. The TTX capillary was then removed about $12 \mathrm{hr}$ prior to the experiment. By the time of anesthesia for the experiment, the toe-spreading reflex on the left side had already recovered. Thus, monosynaptic EPSPs could be recorded from spinal motoneurons in response to stimulation of muscle nerves distal to the site of conduction block. Since the length of the sciatic nerve central to the site of nerve block was too short to stimulate, no attempts were made to assure the absence of muscle contractions in response to electrical stimulation of the nerve during the application of the TTX capillary (see Czéh et al., 1978; Gallego et al., 1979). However, in our previous study in which the effectiveness of TTX capillaries was also assessed by the absence of the toe-spreading reflex, about $86 \%$ of motor nerve terminals examined in the hind leg muscle on the nerve- 
MG-stim.

A

Figure 1. Monosynaptic EPSPs recorded from MG motoneurons in a normal rat $(A)$ and a rat whose conduction in the sciatic nerve had been blocked for 2 d with TTX $(B)$. Each record is the average of 10 EPSPs. The antidromic action potentials superimposed on the homonymous EPSPs in response to stimulation of the MG nerve were blocked by hyperpolarizing currents applied through the intracellular electrode, leaving only the M-spikes (first peaks on the left records). The heteronymous EPSPs were elicited by stimulation of the LGS nerve (right records).
LGS-stim.
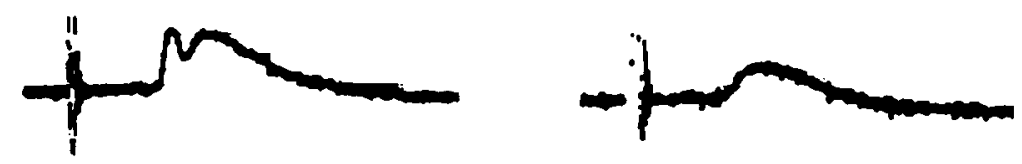

$\mathbf{B}$

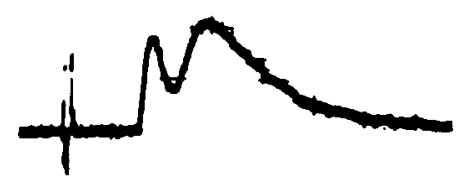

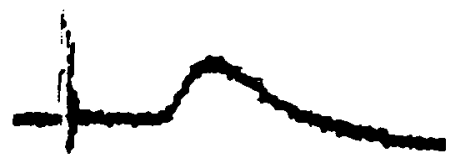

blocked side showed sprouting (Tsujimoto and Kuno, 1988). Therefore, we assumed that the conduction of the majority, if not all, of the nerve fibers in the sciatic nerve would have effectively been blocked by the TTX capillary.

Recording of monosynaptic EPSPs from motoneurons. After chronic conduction block of the left sciatic nerve with TTX, the rat was anesthetized again with pentobarbital sodium $(50 \mathrm{mg} / \mathrm{kg}$, i.p.). During the experiment, one-third the initial dose of pentobarbital was added whenever the corneal reflex of the animal recovered. The procedures used for recording EPSPs were essentially the same as those described previously (Miyata et al., 1986). After tracheostomy, the spinal cord was transected at the midthoracic level, and the lumbar cord was exposed by laminectomy. All the dorsal and ventral roots were left intact in order to record EPSPs from the motoneurons identified by antidromic action potentials in response to stimulation of the hind leg muscle nerves. In the left hind leg, the medial gastrocnemius (MG) and lateral gastrocnemius plus soleus (LGS) nerves were cut distally and prepared for stimulation. Intracellular potentials of motoneurons were recorded with glass microelectrodes filled with $4 \mathrm{M} \mathrm{K}$-acetate. The electrode resistances were between 15 and $25 \mathrm{M}$. Under natural respiration, stable recording from motoneurons was difficult because of movements of the spinal cord. Therefore, natural respiration was blocked by overventilation with an artificial respirator.

Recordings from MG or LGS motoneurons were identified by antidromic action potentials in response to stimulation of the respective muscle nerve. Motoneurons with an action potential of less than $65 \mathrm{mV}$ were discarded. Monosynaptic EPSPs were evoked by stimulation of each of the 2 muscle nerves at a frequency of $0.5 \mathrm{~Hz}$ at an intensity yielding the maximal amplitude. The mean maximal amplitude was obtained by averaging 10 consecutively elicited EPSPs. In the case of homonymous EPSPs (EPSPs evoked in a motoneuron by stimulation of the nerve to the muscle innervated by the motoneuron), the antidromic action potential was often elicited at a stimulus intensity lower than that required to evoke the maximal EPSPs. In these cases, the antidromic action potential was blocked to the M-spike by applying hyperpolarizing currents through the intracellular electrode to dissociate the homonymous EPSP from the antidromic spike (Fig. 1, left records). The applied hyperpolarizing currents did not significantly affect the EPSP amplitude when examined for the heteronymous EPSPs (EPSPs evoked in a motoneuron by stimulation of its synergic muscle nerve) produced in the same motoneuron (also see Gallego et al., 1979). Technical details of this method have already been described (Burke, 1968; Gallego et al., 1979). Hamm et al. (1983) have reported that the maximal amplitude of homonymous EPSPs measured by this method might result in an ovcrestimate by a fraction of a millivolt. This possible crror was not corrected.

Monosynaptic EPSPs were also recorded from age-matched normal, unoperated rats as a control to compare with those observed in the animals exposed to different experimental conditions. In 25 normal, unoperated rats, intracellular potentials were recorded from, in total, 102 MG and 96 LGS motoneurons. Heteronymous EPSPs were observed in all these motoneurons. However, because of difficulties in blocking the antidromic action potential with hyperpolarizing currents (see above), the amplitudes of the homonymous EPSPs could be measured only in $40-55 \%$ of the motoneurons recorded (e.g., Fig. 2). For each experimental group, the homonymous EPSPs were sampled from at least $20 \mathrm{MG}$ and $20 \mathrm{LGS}$ motoneurons. In order to minimize a sample bias from particular animals, no more than 10 MG and 10 LGS motoneurons were recorded from each animal. Thus, the observations were made in at least 5 rats for each experimental group. The statistical analysis was made by 2 -tailed $t$ tests with the significance limit of $p<$ 0.05 unless otherwise stated. In the case in which multiple subgroups were compared under different experimental conditions in addition to nerve block (e.g., varying periods of nerve block or nerve section combined with TTX applications), the analysis of variance was applied, using the variance ratio, $F$, as a test of significance. All values described in the text give means $\pm \mathrm{SD}$. During the experiment, the rectal temperature of the animal was kept at $36.5 \pm 1^{\circ} \mathrm{C}$.

\section{Results}

Time course of changes in the EPSP amplitude by nerve block Figure 1 illustrates some sample records of monosynaptic EPSPs recorded from $\mathrm{MG}$ motoneurons in response to stimulation of the MG (homonymous) or LGS (heteronymous) nerve in a normal, unoperated rat $(A)$ and in a rat in which the conduction of the sciatic nerve had been blocked with TTX for $2 \mathrm{~d}(B)$. As exemplified by these records, both the homonymous and heteronymous EPSPs were generally enhanced after $2 \mathrm{~d}$ of nerve block, compared with those observed in normal, unoperated rats. These results are summarized in Figure 2, in which the heteronymous EPSP amplitude is plotted as a function of the homonymous EPSP amplitude for individual MG $(A)$ or LGS $(B)$ motoneurons recorded from normal rats and after conduction block of the sciatic nerve for $2 \mathrm{~d}$. In agreement with monosynaptic EPSPs recorded from cat motoneurons (Eccles et al., 1957), the homonymous EPSPs were larger than the heteronymous EPSPs in the majority of motoneurons of the rat. Also, the relation between the homonymous and heteronymous EPSP amplitudes was approximately linear in normal rats (Fig. 2, open circles), as was the case in the cat (Burke et al., 1976). Following conduction block of the sciatic nerve for $2 \mathrm{~d}$, the amplitudes of homonymous and heteronymous EPSPs increased approxi- 


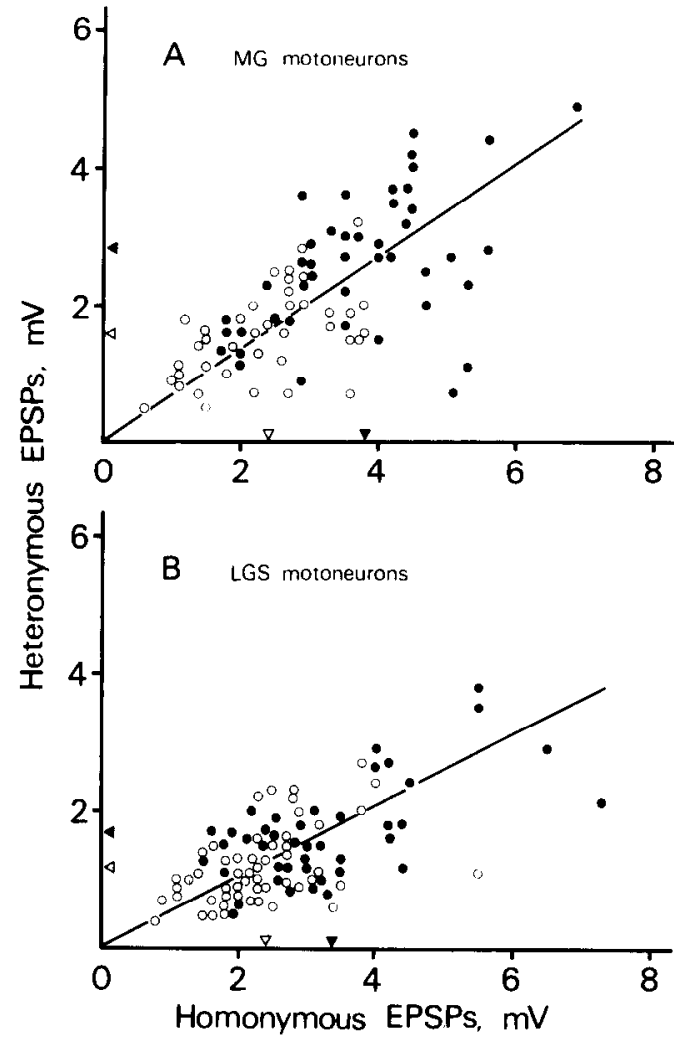

Figure 2. Relations between the homonymous and hetcronymous EPSP amplitudes for individual MG $(A)$ and LGS $(B)$ motoneurons. Open circles, normal, unoperated rats; filled circles, rats in which the conduction of the sciatic nerve had been blocked for $2 \mathrm{~d}$ with TTX. Open and filled triangles on the ordinates and abscissae indicate mean amplitudes. The regression lines were calculated from the results obtained from normal, unoperated rats, based on the assumption of a linear relation.

mately to the same extent, so that these points (Fig. 2, filled circles) also distributed along the same regression line as that for normal EPSPs. The mean amplitudes of EPSPs evoked in MG motoneurons by stimulation of the MG nerve $(2.5 \pm 1.1 \mathrm{mV}$; $n=49)$ and the LGS nerve $(1.4 \pm 0.6 \mathrm{mV} ; n=102)$ in normal rats increased to $3.8 \pm 1.2 \mathrm{mV}(n=38)$ and $2.5 \pm 1.1 \mathrm{mV}(n$ $=69$ ), respectively, after $2 \mathrm{~d}$ of nerve block. Similarly, the mean amplitudes of EPSPs elicited in LGS motoneurons by stimulation of the LGS nerve $(2.3 \pm 0.8 \mathrm{mV} ; n=57)$ and the MG nerve $(1.1 \pm 0.5 \mathrm{mV} ; n=96)$ in normal rats increased to 3.3 $\pm 1.3 \mathrm{mV}(n=29)$ and $1.6 \pm 0.7 \mathrm{mV}(n=54)$, respectively, by the same procedure. All these increments were highly significant $(p<0.001)$.

Among the 4 classes of monosynaptic EPSPs evoked in MG or LGS motoneurons by stimulation of the MG or LGS nerve, the degree of the disuse-induced EPSP enhancement varied from 1.4- to 1.8 -fold (mean, 1.58 -fold). This was greater than the degree of EPSP enhancement observed in motoneurons of the cat following conduction block of the peripheral nerve for 2 weeks, 1.2- to 1.4-fold (Gallego et al., 1979), or than that seen in the sympathetic ganglion after preganglionic block for $2 \mathrm{~d}$, 1.2-fold (Gallego and Geijo, 1987). It might be argued that the increase in the EPSP amplitude observed following chronic nerve block results from alterations in the membrane properties of motoneurons. However, the mean input resistance $(1.57 \pm 0.31$ $\mathrm{M} \Omega$ ) mcasurcd by the spike height method (Frank and Fuortes, 1956) for MG and LGS motoneurons $(n=48)$ in normal, un-

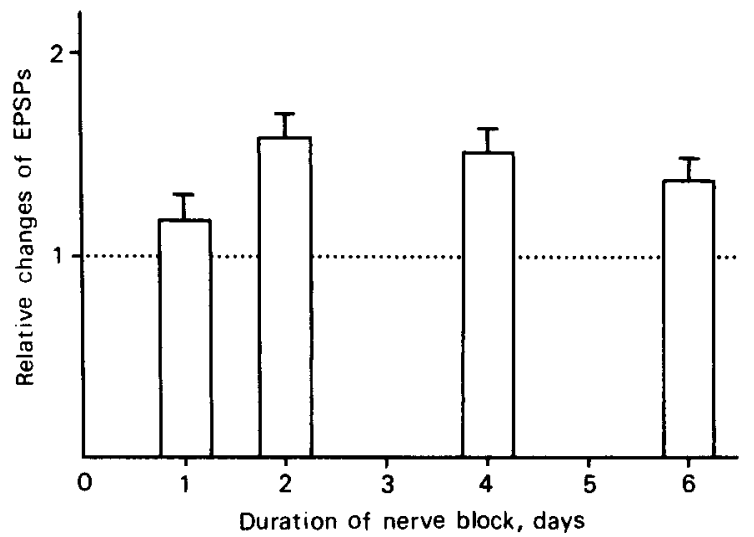

Figure 3. Degree of synaptic enhancement as a function of the period of conduction block of the sciatic nerve with TTX. Each column represents the mean normalized EPSP amplitude cxpressed in terms of individual EPSP amplitudes relative to the average corresponding EPSP amplitude observed in normal, unoperated rats, taking the latter value as unity. Horizontal bars, 2 SEM.

operated rats was not significantly $(0.4<p<0.5)$ different from that $(1.52 \pm 0.23 \mathrm{M} \Omega ; n=28)$ observed after nerve block for $2 \mathrm{~d}$. The input resistance distributions between the 2 groups were also indistinguishable in their ranges (1.0-2.2 and 1.0-1.9 $\mathrm{M} \Omega$ ) and medians (1.6 and $1.5 \mathrm{M} \Omega$ ). In addition, the mean resting potential of MG and LGS motoneurons in normal rats $(-59.4 \pm 7.2 \mathrm{mV} ; n=43)$ was not significantly $(0.4<p<0.5)$ different from that in the experimental animals $(-58.4 \pm 6.2$ $\mathrm{mV} ; n=48)$. Again, their ranges $(-75--50$ and $-75--50 \mathrm{mV})$ and medians $(-60$ and $-58 \mathrm{mV})$ were comparable. Furthermore, implantation of a control capillary filled with $0.9 \% \mathrm{NaCl}$ alone (without TTX) even for $4 \mathrm{~d}$ did not induce any significant changes in the homonymous and heteronymous EPSP amplitudes ( $90.8 \%$ of the normalized control value; see below) of 29 MG and 32 LGS motoneurons. However, Gallego et al. (1979) have reported that some reduction (3-14\%) in the size of afferent volleys occurred after the chronic application of a TTX cuff to the peripheral nerve in the cat. The tendency of a slight reduction in the mean EPSP amplitude observed after implantation of the control capillary, although insignificant, might have reflected the possible injuries of a few Ia sensory fibers induced by the capillary.

Figure 3 shows changes in the mean monosynaptic EPSP amplitude following conduction block of the sciatic nerve for $1-6 \mathrm{~d}$. In this figure, the 4 classes of monosynaptic EPSPs evoked in MG or LGS motoneurons by stimulation of the MG or LGS nerve were normalized and expressed in terms of relative changes of the EPSP amplitude, taking the mean amplitude of each class of the EPSPs observed in normal, unoperated rats as unity. The EPSPs were significantly enhanced following conduction block of the peripheral nerve even for $1 \mathrm{~d}(p<0.05, F$ ratio test), and the maximal enhancement was obtained after $2 \mathrm{~d}$ of nerve block (Fig. 3). There was no tendency of a further increase in the EPSP amplitude when the period of conduction block had been prolonged to 4 or $6 \mathrm{~d}$. In fact, the magnitude of synaptic enhancement seen after $6 \mathrm{~d}$ of conduction block was slightly but significantly ( $p<0.01, F$ ratio test) less than that induced by a 2 d block (Fig. 3). It is not certain whether this small change is genuine. However, it secms clcar that plastic changes of synaptic function induced by disuse are fully expressed within $2 \mathrm{~d}$. Again, 
the mean input resistance of motoneurons after 1,4 , or $6 \mathrm{~d}$ of nerve block was comparable to the normal value.

\section{Early enhancement of EPSPs following peripheral nerve} section

Miyata and Yasuda (1988) have shown that monosynaptic EPSPs recorded from rat motoneurons are potentiated during the first few days after section of the peripheral muscle nerve. We have examined whether this phenomenon can be accounted for entirely by elimination of the sensory impulse activity. For this purpose, only the MG nerve was cut near the muscle at the time when a TTX capillary was implanted to the sciatic nerve. Spontaneous impulse activity may arise from a neuroma formed at the cut end of the muscle nerve (Wall and Gutnick, 1974; Blumberg and Janig, 1984). However, the possible spontaneous discharge arising from a neuroma would have been blocked by the TTX capillary placed in the sciatic nerve central to the cut MG nerve. Therefore, if there is no additional effect induced by section of the MG nerve, one would expect to obtain the same relationship between the homonymous and heteronymous EPSP amplitudes after a $2 \mathrm{~d}$ block as that shown in Figure 2 (filled circles). In fact, monosynaptic EPSPs evoked by stimulation of the MG nerve as well as by stimulation of the LGS nerve were significantly ( $p<0.001, F$ ratio test) enhanced under these conditions (compare open circles in Figs. 2 and 4). Unexpectedly, however, section of the MG nerve caused a greater increase in the amplitude of monosynaptic EPSPs evoked by stimulation of the MG nerve. As shown in Figure 4 (open circles), when section of the MG nerve was combined with a $2 \mathrm{~d}$ block of the sciatic nerve, the distribution of the points deviated from the expected regression lines, showing disproportionate enhancement of EPSPs elicited by stimulation of the cut MG nerve: 1.34 times greater than the degree of enhancement of EPSPs evoked by stimulation of the LGS nerve, a significant difference ( $p<0.01, F$ ratio test). The mean input resistance of MG motoneurons (1.93 $\pm 0.43 \mathrm{M} \Omega$; range, $1.3-2.8 \mathrm{M} \Omega ; n=10$ ) increased significantly $(p<0.01) 2 \mathrm{~d}$ after nerve section compared with that observed following nerve block alone $(1.51 \pm$ $0.25 \mathrm{M} \Omega$; range, $1.0-1.9 \mathrm{M} \Omega ; n=20$ : also see Miyata and Yasuda, 1988). Therefore, an increase of EPSP amplitudes in MG motoneurons might be attributed in part to increased input resistance. However, this factor cannot account for an increase in the amplitude of heteronymous EPSPs evoked in intact LGS motoneurons by stimulation of the cut MG nerve (Fig. $4 B$ ).

From the above results it seems clear that section of the MG nerve induces surplus enhancement of central synaptic function in addition to the effect of elimination of sensory impulse activity by TTX. However, it should be noted that, when the MG nerve is sectioned, the sensory impulse activity in the MG nerve remains absent after removal of the TTX capillary $12 \mathrm{hr}$ before the experiment. Thus, it is possible that the absence of resumption of sensory impulse activity during the $12 \mathrm{hr}$ following removal of the TTX capillary may account for the surplus enhancement of central synaptic function. In order to test this possibility, the conduction of the sciatic nerve had first been blocked with TTX for $2 \mathrm{~d}$, and when the TTX capillary was removed $12 \mathrm{hr}$ before the experiment, the MG nerve was sectioned near the muscle. Under this condition, sensory impulse activity from the LGS muscle recovers during about $12 \mathrm{hr}$ after removal of the TTX capillary prior to the experiment, whereas sensory impulse activity in the MG nerve blocked by TTX remains silent during the $12 \mathrm{hr}$ period because of additional section. Figure 4 (filled circles) shows the results obtained from this series of experiments. In these experiments, the mean amplitudes of monosynaptic EPSPs elicited by stimulation of the MG nerve and the LGS nerve were both greater than those observed in normal animals ( $p<0.001, F$ ratio test). However, the EPSPs evoked in MG $(A)$ or LGS $(B)$ motoneurons by stimulation of the $\mathrm{MG}$ nerve were again disproportionately enhanced (1.42 times greater than the degree of enhancement of EPSPs elicited by stimulation of the LGS nerve; this difference was significant at $p<0.01, F$ ratio test). Thus, synaptic enhancement observed $2.5 \mathrm{~d}$ after section of the MG nerve could be mimicked by conduction block of the muscle nerve with TTX for $2 \mathrm{~d}$, if resumption of the sensory impulse activity during the $12 \mathrm{hr}$ period after removal of the TTX capillary was prevented by additional nerve section.

In order to test whether central synaptic efficacy might be affected by the additional nerve section alone, monosynaptic EPSPs were recorded $12 \mathrm{hr}$ after section of the MG nerve without TTX treatment. Under this condition, the mean amplitudes of homonymous and heteronymous EPSPs evoked in $37 \mathrm{MG}$ and 31 LGS motoneurons by stimulation of the cut MG nerve showed no significant difference from those observed in normal, unoperated rats $(108.7 \%$ of the normalized, control value). Thus, the early potentiation of monosynaptic EPSPs in motoneurons produced by section of the muscle nerve can be accounted for entirely by the effect of elimination of sensory impulse activity from the muscle.

\section{Recovery of disuse-induced synaptic enhancement}

The above results imply that synaptic enhancement once acquired by a $2 \mathrm{~d}$ block of the sensory input activity declines substantially within about $12 \mathrm{hr}$ after resumption of sensory impulse activity. One might then ask how long the acquisition of disuse-induced synaptic enhancement may be retained. To address this question, monosynaptic EPSPs were recorded 48 and $72 \mathrm{hr}$ after removal of the TTX capillary that had been applied to the sciatic nerve for $2 \mathrm{~d}$. Figure 5 shows the mean amplitudes of monosynaptic EPSPs produced in MG and LGS motoneurons by stimulation of the $\mathrm{MG}$ nerve after resumption of natural sensory impulse activity. The maximal synaptic enhancement at time 0 shows the results obtained from the animals whose MG nerves had been blocked with TTX for $2 \mathrm{~d}$ and sectioned at the time when the TTX capillary was removed 12 hr prior to the experiment (see filled circles in Fig. 4). When natural resumption of the MG sensory activity had been allowed during the $12 \mathrm{hr}$ period following removal of the TTX capillary (without section of the MG nerve), the degree of synaptic enhancement declined by about $45 \%$ (Fig. 5 , points at $12 \mathrm{hr}$ ). This decrement was significant ( $p<0.05, F$ ratio test). However, further extinction of synaptic enhancement occurred slowly, and synaptic function remained significantly elevated $(p<0.05, F$ ratio test) for at least $3 \mathrm{~d}$ after resumption of the impulse activity.

Miyata and Yasuda (1988) have shown that the early potentiation of monosynaptic EPSPs in rat motoneurons following section of the muscle nerve can be prevented by treatment with actinomycin D(AMD). This suggests that the synthesis of some macromolecules is required for the expression of synaptic enhancement. In about 20 rats, we injected AMD intraperitoneally at a dose of $0.4 \mathrm{mg} / \mathrm{kg}$ (see Grampp et al., 1972) immediately before section of the MG nerve, and attempts were made to record monosynaptic EPSPs 2 d later. However, all animals treated with AMD expired before or during dissection for the 


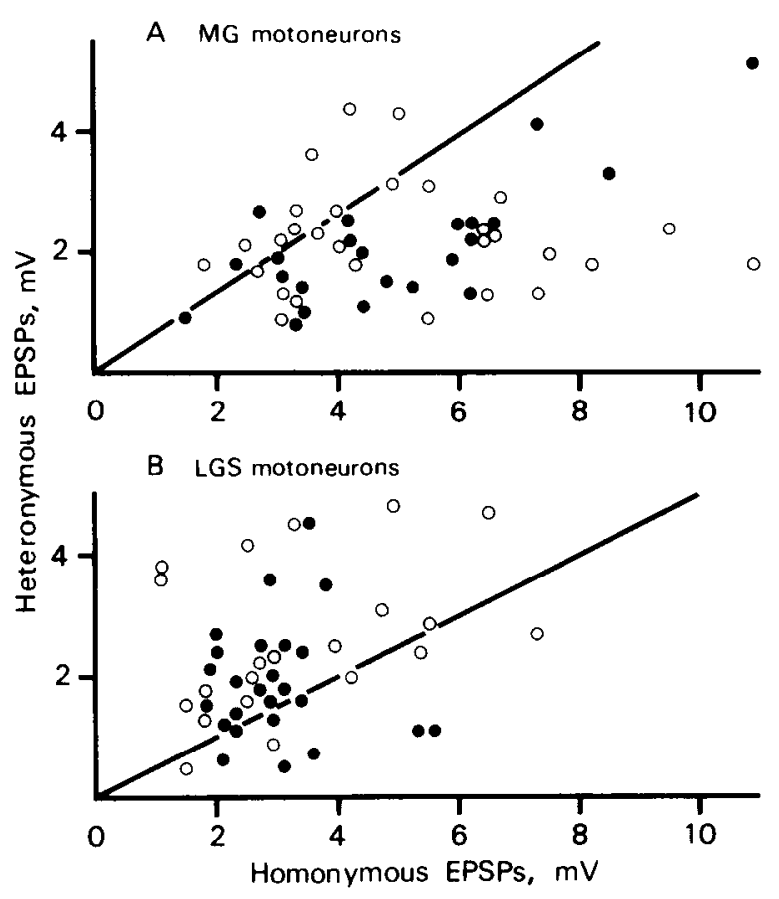

Figure 4. Relationships between the homonymous and heteronymous EPSP amplitudes in MG $(A)$ and LGS $(B)$ motoneurons. Open circles, rats in which the MG nerve had been sectioned when a TTX capillary was implanted to the sciatic nerve to block its conduction for $2 \mathrm{~d}$; filled circles, rats in which the MG nerve had been sectioned when the TTX capillary implanted to the sciatic nerve for $2 \mathrm{~d}$ was removed about 12 hr prior to the experiment. The regression lines were reproduced from those shown in Figure 2.

experiment. Similarly, a number of rats in which nerve conduction had been blocked with TTX also succumbed to drug toxicity.

\section{Discussion}

The present results have confirmed that chronic block of the sensory input to the spinal cord causes enhancement of Ia synaptic transmission in spinal motoneurons (Gallego et al., 1979, 1980). Synaptic enhancement was found to be expressed fully within $2 \mathrm{~d}$ of nerve block. This is in contrast with a similar disuse-induced enhancement in Ia synaptic transmission observed after 2 weeks of conduction block of the peripheral nerve in the cat (Gallego et al., 1979). However, since the effects of different periods of nerve block have not been examined in motoneurons of the cat, it is not certain whether the period of disuse necessary for the acquisition of synaptic enhancement in motoneurons differs in the 2 animal species.

The mechanism of synaptic enhancement induced by chronic block of the sensory input remains elusive. It has been suggested that this synaptic enhancement might be due to an increase in sensitivity of the postsynaptic receptors for the transmitter, being analogous to "denervation supersensitivity" (Gallego et al., 1979; Mendell, 1984) or to central terminal sprouting of Ia sensory fibers (Gallego et al., 1979). At neuromuscular junctions, denervation supersensitivity can be induced $3 \mathrm{~d}$ after conduction block of the motor nerve (Lomo and Rosenthal, 1972). Similarly, the sprouting of motor nerve terminals occurs when conduction of the motor nerve is blocked with TTX for more than $3.5 \mathrm{~d}$ (Brown and Ironton, 1977). The period of nerve block required for the expression of central synaptic enhancement (1-

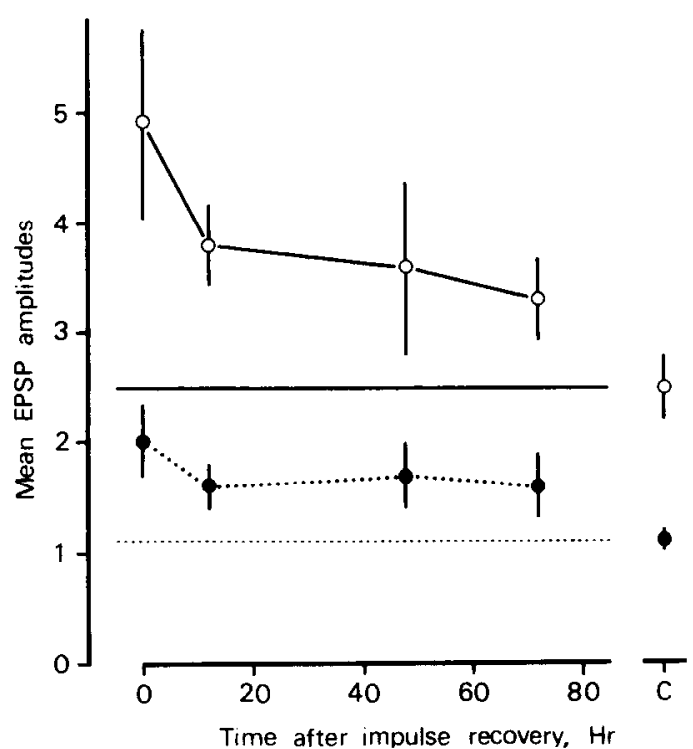

Figure 5. Degree of synaptic enhancement induced by conduction block of the sciatic nerve for $2 \mathrm{~d}$ with TTX as a function of the time after resumption of sensory impulse activity. Open circles, mean amplitudes of EPSPs evoked in MG motoneurons by stimulation of the MG nerve; filled circles, mean amplitudes of EPSPs evoked in LGS motoneurons by stimulation of the MG nerve. Vertical bars, 2 SEM. Horizontal solid line and open circle at $C$ indicate the mean amplitude of EPSPs produced in MG motoncurons by stimulation of the MG nerve observed in normal, unoperated rats. Broken horizontal line and flled circle at $C$ similarly indicate the control mean value of EPSPs evoked in LGS motoneurons by stimulation of the MG nerve. Ordinate, $\mathrm{mV}$.

2 d) appears to be too short to consider these phenomena as the underlying mechanisms. Also, it has recently been shown that disuse-induced enhancement in transmission at neuromuscular junctions is not necessarily accompanied by terminal sprouting (Tsujimoto and Kuno, 1988). Currently, however, no information is available for the time course of induction of the possible denervation supersensitivity or terminal sprouting in the CNS. Enhancement of monosynaptic spinal reflexes evoked by afferent volleys from chronically tenotomized muscles in the cat has been suggested to result from prolonged disuse of the Ia sensory pathway (Beránek and Hnik, 1959). This enhanced reflex was observed for several weeks after tenotomy but not in the longer (18-80 weeks) postoperative periods (Beránek et al., 1961). A similar recovery of enhanced synaptic function to normal levels has also been noted during 20-30 weeks of disuse conditions induced by different experimental procedures (Gallego et al., 1980; Mayer et al., 1984; also see Nelson and Mendell, 1979). It is thus possible that central synaptic enhancement once induced under disuse conditions may be subject to long-term adaptation.

The disuse-induced synaptic enhancement observed in the present study can be maintained for at least $3 \mathrm{~d}$ after resumption of the sensory impulse activity. In this sense, this is apparently a long-term plasticity. The long-term synaptic facilitation (Montarolo et al., 1986) as well as the long-term heterosynaptic inhibition (Montarolo et al., 1988) observed in Aplysia requires the synthesis of new macromolecules, whereas development of the short-term synaptic plasticity is not affected by treatment with an inhibitor of protein synthesis. Similarly, the late phase of the long-term potentiation in the hippocampus can be blocked 
by an inhibitor of protein synthesis (Frey et al., 1988). Gallego and Geijo (1987) have found that chronic conduction block of preganglionic fibers between the superior cervical ganglion (SCG) and the stellate ganglion causes synaptic enhancement in the stellate ganglion proximal to the site of conduction block as well as in the distal SCG. These results suggest the participation of some changes in the cell bodies of preganglionic neurons in the expression of disuse-induced synaptic enhancement in the sympathetic ganglion. In fact, Miyata and Yasuda (1988) could prevent the early potentiation of Ia EPSPs following section of the peripheral nerve by treatment with AMD, although we were not able to reproduce these results because of the drug toxicity. It is conceivable that prolonged elimination of impulse activity in sensory fibers leads to the synthesis of some macromolecules in the cell bodies of sensory neurons, which, in turn, triggers enhancement of central synaptic function of the sensory neurons. This situation may be considered analogous to a striking increase of substance $P$ or its messenger RNA induced in cultured sympathetic ganglion cells by prolonged elimination of electrical activity with TTX (Black et al., 1987).

The degree of enhancement of Ia EPSPs induced by chronic conduction block of the peripheral nerve was comparable to that observed a few days after section of the peripheral nerve. Also, the minimal period of nerve block ( $24 \mathrm{hr}$ or less) and that following nerve section (between 12 and $24 \mathrm{hr}$ ) necessary for the acquisition of central synaptic enhancement were indistinguishable. Therefore, it seems reasonable to conclude that the early potentiation of Ia EPSPs following section of the peripheral nerve is due to the effects of elimination of the sensory impulse activity. However, the mean EPSP amplitude initially increased subsides to near-normal levels $4-7 \mathrm{~d}$ after nerve section and is eventually depressed to about $50 \%$ of the control value during the next 1 or 2 weeks (Miyata and Yasuda, 1988). In contrast, a significant synaptic enhancement is still maintained after $6 \mathrm{~d}$ of conduction block of the sciatic nerve. Therefore, it is clear that section of the peripheral nerve gives rise to a slow "delcterious" effect on central synaptic function, in addition to the initial synaptic enhancement due to the deprivation of sensory impulse activity. Synaptic depression in motoneurons produced by peripheral nerve crush in neonatal rats has been found to be reversed by daily treatment with nerve growth factor (NGF; Kuno et al., 1985; Miyata et al., 1986). Thus, central synaptic depression observed following peripheral nerve injuries may be assumed to be due to interruption of the retrograde supply of NGF or an analogous trophic factor to the sensory neurons from the target muscle (Miyata et al., 1986; Kuno, 1987). Recently, the cell death of NGF-deprived sympathetic neurons has been shown to be prevented by inhibiting protein or RNA synthesis (Martin et al., 1988). This suggests that the deprivation of the trophic factor may cause the synthesis of particular macromolecules that are "deleterious" to the neurons. Whatever the undcrlying mechanism, it is now concluded that sensory impulse activity and the peripheral axonal continuity of sensory fibers are 2 distinct factors in the maintenance of central synaptic function.

\section{References}

Beránek, R., and P. Hnik (1959) Long-term effects of tenotomy on spinal monosynaptic response in the cat. Science 130:981-982.

Beránek, R., P. Hnik, L. Vyklický, and J. Zelená (1961) Facilitation of the monosynaptic spinal reflex due to long-term tenotomy. Physiol. Bohemoslov. 10: 543-552.

Black, I. B., J. E. Adler, C. F. Dreyfus, W. F. Friedman, E. F. LaGamma, and A. H. Roach (1987) Biochemistry of information storage in the nervous system. Science 236: 1263-1268.

Blumberg, H., and W. Janig (1984) Discharge pattern of afferent fibers from a neuroma. Pain 20: 335-353.

Brown, M. C., and R. Ironton (1977) Motor neurone sprouting induced by prolonged tetrodotoxin block of nerve action potentials. Nature 265: 459-461.

Burke, R. E. (1968) Group Ia synaptic input to fast and slow twitch motor units of cat triceps surae. J. Physiol. (Lond.) 196: 605-630.

Burke, R. E., W. Z. Rymer, and J. V. Walsh (1976) Relative strength of synaptic input from short-latency pathways to motor units of defined type in cat medial gastrocnemius. J. Neurophysiol. 39: 447458.

Czéh, G., R. Gallego, N. Kudo, and M. Kuno (1978) Evidence for the maintenance of motoneurone properties by muscle activity. J. Physiol. (Lond.) 281: 239-252.

Eccles, J. C., R. M. Eccles, and A. Lundberg (1957) The convergence of monosynaptic excitatory afferents on to many different species of alpha motoneurones. J. Physiol. (Lond.) 137: 22-50.

Eccles, J. C., K. Krnjević, and R. Miledi (1959) Delayed effects of peripheral severance of afferent nerve fibres on the efficacy of their central synapses. J. Physiol. (Lond.) 145: 204-220.

Frank, K., and M. G. F. Fuortes (1956) Stimulation of spinal motoneurones with intracellular electrodes. J. Physiol. (Lond.) 134: 451470.

Frey, U., M. Krug, K. G. Reymann, and H. Matthies (1988) Anisomycin, an inhibitor of protein synthesis, blocks late phase of LTP phenomena in the hippocampal CA1 region in vitro. Brain Res. 452: $57-65$.

Gallego, R., and E. Geijo (1987) Chronic block of the cervical trunk increases synaptic efficacy in the superior and stellate ganglia of the guinea-pig. J. Physiol. (Lond.) 382: 449-462.

Gallego, R., M. Kuno, R. Núñez, and W. D. Snider (1979) Disuse enhances synaptic efficacy in spinal motoneurones. J. Physiol. (Lond.) 291: 191-205.

Gallego, R., M. Kuno, R. Núñez, and W. D. Snider (1980) Enhancement of synaptic function in cat motoneurones during peripheral sensory regeneration. J. Physiol. (Lond.) 306: 205-218.

Goldring, J. M., M. Kuno, R. Núñez, and W. D. Snider (1980) Reaction of synapses on motoneurones to section and restoration of peripheral sensory connexions in the cat. J. Physiol. (Lond.) 309: 185198

Grampp, W., J. B. Harris, and S. Thesleff (1972) Inhibition of denervation changes in skeletal muscle by blockers of protein synthesis. J. Physiol. (Lond.) 221: 743-754.

Hamm, T. M., B. R. Botterman, R. M. Reinking, and D. G. Stuart (1983) Characteristics of $M$ spikes in cat motoneurons and their significance for the measurement of small composite Ia EPSPs. Exp. Brain Res. 49: 68-76.

Kuno, M. (1987) Enhancement of central synaptic efficacy by prolonged disuse of the synapse. In Strategy and Prospects in Neuroscience, O. Hayaishi, ed., pp. 187-198, Japan Science Society, Tokyo.

Kuno, M., Y. Miyata, S. Homma, and M. Ogawa (1985) Nerve growth factor enhances central synaptic function of Ia sensory neuron. Neurosci. Res. 2: 275-280.

Lømo, T., and J. Rosenthal (1972) Control of ACh sensitivity by muscle activity in the rat. J. Physiol. (Lond.) 221: 493-513.

Martin, D. P., R. E. Schmidt, P. S. DiStefano, O. H. Lowry, J. G. Carter, and E. M. Johnson (1988) Inhibitors of protein synthesis and RNA synthesis prevent neuronal death caused by nerve growth factor deprivation. J. Cell Biol. 106: 829-844.

Mayer, R. F., R. E. Burke, J. Toop, B. Walmsley, and J. A. Hodgson (1984) The effect of spinal cord transection on motor units in cat medial gastrocnemius muscles. Muscle Nerve 7: 23-31.

Mendell, L. M. (1984) Modifiability of spinal synapses. Physiol. Rev. 64: 260-324.

Mills, R. G., and J. J. Bray (1979) A slow-release technique for inducing prolonged paralysis by tetrodotoxin. Pfluegers Arch. 383: 6774.

Miyata, Y., and H. Yasuda (1988) Enhancement of Ia synaptic transmission following nerve section: Dependence upon protein synthesis. Neurosci. Res. 5: 338-346.

Miyata, Y., Y. Kashihara, S. Homma, and M. Kuno (1986) Effects of nerve growth factor on the survival and synaptic function of Ia sensory neurons axotomized in neonatal rats. J. Neurosci. 6: 2012-2018.

Montarolo, P. G., P. Goelet, V. F. Castellucci, J. Morgan, E. R. Kandel, 
and S. Schacher (1986) A critical period for macromolecular synthesis in long-term heterosynaptic facilitation in Aplysia. Science 234: $1249-1254$.

Montarolo, P. G., E. R. Kandel, and S. Schacher (1988) Long-term heterosynaptic inhibition in Aplysia. Nature 333: 171-174.

Nelson, S. G., and L. M. Mendell (1979) Enhancement in Ia motoneuron synaptic transmission caudal to chronic spinal cord transection. J. Neurophysiol. 42: 642-654.
Tsujimoto, T., and M. Kuno (1988) Calcitonin gene-related peptide prevents disuse-induced sprouting of rat motor nerve terminals. J. Neurosci. 8: 3951-3957.

Wall, P. D., and M. Gutnick (1974) Ongoing activity in peripheral nerves: The physiology and pharmacology of impulses originating from a neuroma. Exp. Neurol. 43: 580-593. 